\title{
Left Ventricular "Horseshoe-Thrombus"- A Case Report
}

\author{
Ramachandran Muthiah \\ Thoothukudi Medical College Hospital, Thoothukudi, India \\ Email: cardioramachandran@yahoo.co.uk
}

Received 10 March 2016; accepted 23 April 2016; published 26 April 2016

Copyright (C) 2016 by author and Scientific Research Publishing Inc.

This work is licensed under the Creative Commons Attribution International License (CC BY). http://creativecommons.org/licenses/by/4.0/

(c) () Open Access

\section{Abstract}

Thrombi represent the most frequently found intracardiac masses. Left ventricular thrombus (LVT) is an important complication in patients with ischemic heart diseases and in those with dilated cardiomyopathy and systolic heart failure. The diagnosis of left ventricular thrombus remains important since anticoagulation will reduce the risk of systemic embolization and stroke. Despite advances in other imaging modalities, echocardiography remains the most important tool for diagnosis and risk stratification in patients predisposed to develop left ventricular thrombi. Mural thrombi formed on the left ventricular endocardium and horseshoe-shaped in chronic dilated cardiomyopathy, masquerading as left ventricular endomyocardial fibrosis was diagnosed by transthoracic 2D echocardiographic imaging in a 38 years old middle aged man. Background of this case highlighted the pathophysiology, high risk echocardiographic features and the role of anticoagulant therapy in LV thrombus with dilated cardiomyopathy.

\section{Keywords}

Thrombus, Left Ventricle, Dilated Cardiomyopathy, Echocardiography, Anticoagulant Therapy

\section{Introduction}

Thrombus is defined as a discrete echo dense mass with defined margin, distinct from the endocardium and seen throughout systole and diastole. Intracardiac thrombi are classified as left ventricular, left atrial, right sided and valvular thrombi. Left ventricular thrombi are typically located in areas of severe wall motion abnormalities.

The pathophysiologic mechanism for left ventricular thrombus (LVT) formation in the so-called "Virchow's triad" was postulated in patients with acute myocardial infarction or in those with dilated cardiomyopathy and congestive heart failure. The triad consists of stasis of blood, endothelial injury or dysfunction and a hypercoagulable state. LV regional wall akinesia and dyskinesia result in blood stasis; prolonged ischemia leads to sub- 
endocardial tissue injury with inflammatory changes and a hypercoagulable state. Thus the combination of blood stasis, endothelial injury and hypercoagulability, often referred to as Virchow's triad, is a prerequisite for in vivo thrombus, composed of fibrin, red blood cells, and platelets. Thrombi occupying in heart chambers are designated as mural thrombi. Abnormal myocardial contraction or endocardial injury promotes cardiac mural thrombi formation. In patients with dilated cardiomyopathy, low-velocity swirling of blood within the left ventricle also predisposes to the development of a thrombus due to altered hemodynamics associated with poor myocardial contractility. Thrombi may undergo propagation, embolization and dissolution. The older thrombi become organized by the growth of endothelial cells, smooth muscle cells and fibroblasts. Eventually, with remodeling and contraction of the mesenchymal elements, only a fibrous lump may remain to mark the original thrombus. The fibrosed thrombi may undergo hyalinization and calcification.

It has been speculated that LV thrombus provides a positive role by offering mechanical support to the myocardium and therefore protecting against LV rupture. The thrombus becomes firmly attached to its site of origin, enhancing the underlying myocardial scar, partially restoring the thickness of the myocardial wall and limiting the potential of its expansion.

A chronic fibrosed organized thrombi visible as horseshoe-shaped in LV cavity around the apex in chronic dilated cardiomyopathy is uncommon and so this case had been reported.

\section{Case Report}

A 38 years old middle aged man, having frequent episodes of bronchial asthma for one year duration was referred to this hospital for echocardiography. His pulse rate was $116 \mathrm{bpm}$ and blood pressure 110/70 mmHg. Physical examination revealed normal JVP (Jugular Venous Pressure), enlarged apical impulse, muffled heart sounds and no murmur. Lung fields revealed basal rales and rhonchi. Blood chemistry revealed no abnormalities. ECG was normal. X-ray chest revealed cardiomegaly. Transthoracic 2D echocardiography revealed dilated cardiomyopathy with a measured ejection fraction of $23 \%$ and a chronic, laminar, flat, fibrosed, organized, large horseshoe-shaped thrombus occupying the apex and adjacent portion of LV (left ventricular) cavity as shown in Figure 1, Figure 2 and Figure 3. Figure 1 revealed the horseshoe-shaped thrombus around the LV apex in apical four chamber view. Figure 2 revealed its maximal thickness of $18 \mathrm{~mm}$ in tilted two chamber view, Figure 3 revealed the thrombus embedded along the papillary muscle and a dilated LV cavity of $68 \mathrm{~mm}$ in parasternal long axis view. Color Doppler showed no regurgitant lesions across the AV valves and both mitral and tricuspid leaflets appeared normal.

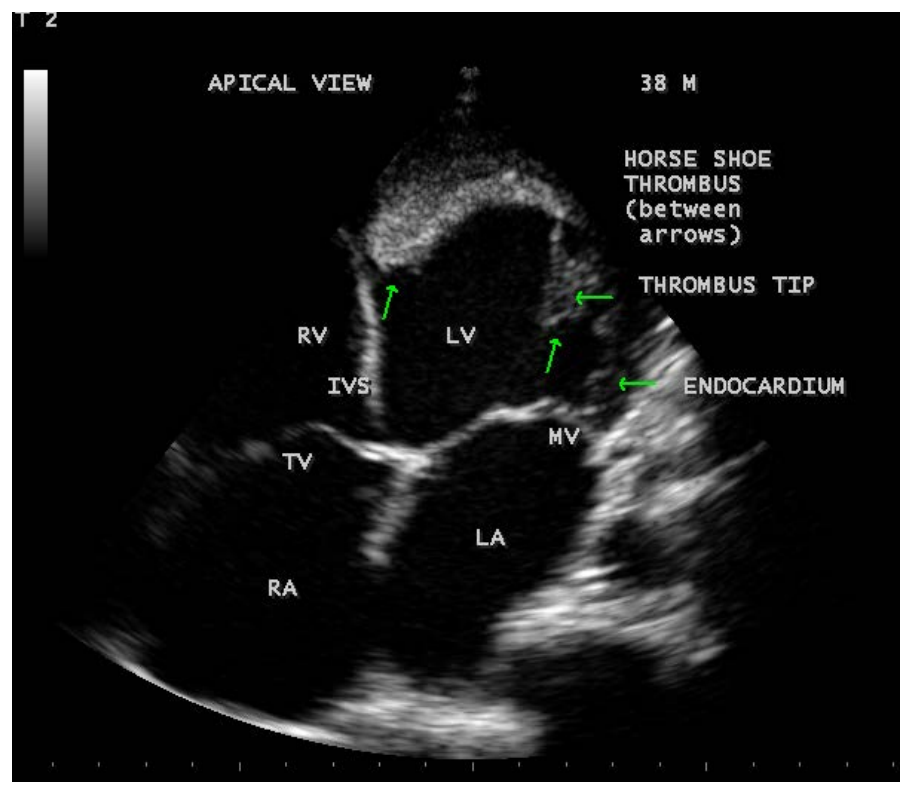

Figure 1. Apical four chamber view showing the horseshoe-shaped thrombus and dilated cardiac chambers suggesting dilated cardiomyopathy. 


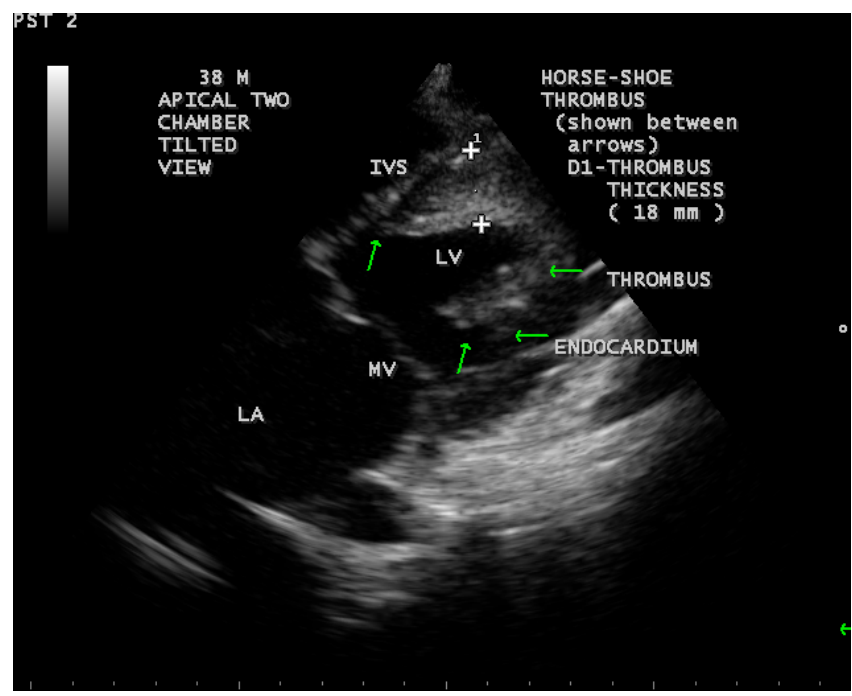

Figure 2. Tilted two chamber view showing the maximal thrombus thickness of $18 \mathrm{~mm}$.

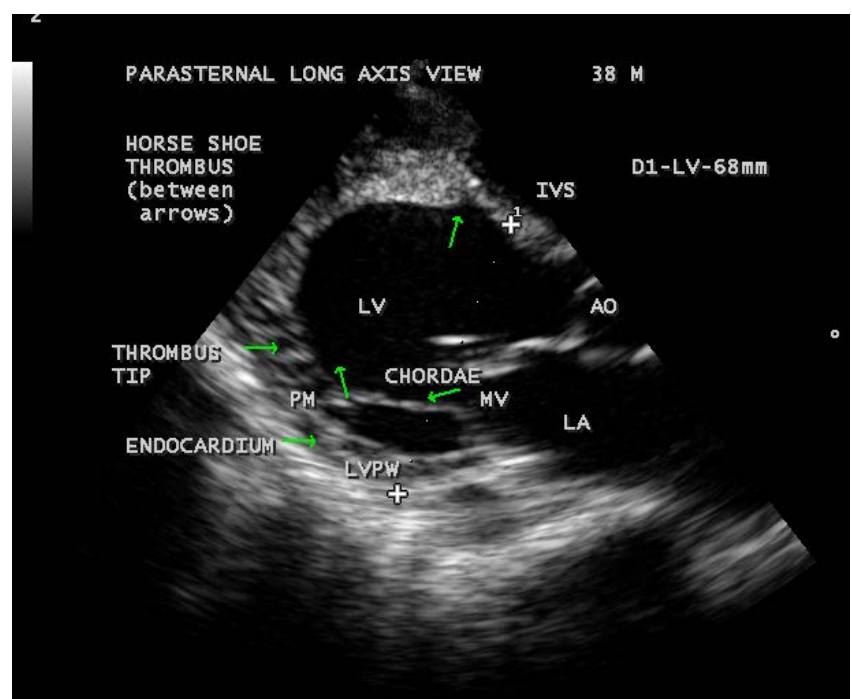

Figure 3. Parasternal long axis view showing the thrombus embedded on the papillary muscle and a dilated LV cavity of $68 \mathrm{~mm}$.

He was treated with antibiotics, bronchodilators and anti-failure measures. He was given a short course of warfarin therapy for one month and no change in the thrombus was observed. Since he developed one or two episodes of mild hemoptysis, warfarin therapy was withdrawn. He developed no embolic events on further follow up of 3 months without warfarin therapy. He died due to acute respiratory failure following a severe asthmatic episode.

\section{Discussion}

\subsection{Frequency}

Previously, the incidence of left ventricular thrombus (LVT) was reported to be as high as $30 \%-40 \%$ in patients with an anterior wall myocardial infarction. For patients with an non-anterior myocardial infarction, the risk of LVT was lower $(<5 \%)$ [1]. Although controversial, in the contemporary era of routine early revascularization and more aggressive anticoagulation, the incidence of LVT complicating an anterior myocardial AMI (acute myocardial infarction) is likely reduced and is currently estimated at 5\% - 15\% [2]-[4]. In patients with dilated 
cardiomyopathy and congestive heart failure (CHF), the reported frequency of LVT varies from $10 \%-30 \%$, depending on the series [5] [6].

\subsection{General Features}

Thrombi are typically amorphous echogenic structures with varying shape and are adherent to the endocardium. Thrombi may be multiple, mobile and may protrude into the left ventricular cavity. In most cases, they have a texture and appearance that are distinct from the adjacent myocardium. Thrombi generally involve the apex of the left ventricle, most often in the presence of akinesis or dyskinesis. Predisposing factors include recent myocardial infarction, left ventricular aneurysm and dilated cardiomyopathy and thrombi can develop in any situation in which low flow and blood stasis occur.

Left ventricular thrombi (LVT) that complicate dilated cardiomyopathy (DCM) also are located more commonly at the apex, perhaps reflecting the propensity for left ventricular stasis, to be located further from the inflow and outflow tracts. Hypercoagulabilty and endothelial dysfunction also, are associated with DCM, fulfilling Virchow's triad [7]. Unlike acute myocardial infarction, however, the development of LVT in patients with DCM is not marked by a distinct clinical event. This may account for the fact that most thrombi are found incidentally during echocardiographic assessment of left ventricular function or during evaluation of an embolic event.

Sometimes the thrombi may be flat lying along the left ventricular wall even in some cases, it may be very difficult to differentiate thrombus from myocardium. The distinction between thrombus and pannus formation is essential. Thrombi in general larger and have soft ultrasound density similar to myocardium, but pannus formation is usually more dense and small in appearance.

\subsection{Echocardiography}

Transthoracic echocardiography remains the most important imaging modality to make a diagnosis of left ventricular thrombus (LVT). Echocardiographic appearance of intracardiac thrombus is heterogenous, they can vary from a small, immobile mural thrombus to a large protruding mobile thrombi. Echodensity and shape of the thrombi depend on age and degree of thrombus organization. They may be homogenously echogenic or may have heterogenous texture with lucent areas. An echolucent center suggest that the thrombus is relatively new and actively growing. Very fresh thrombi tend to protrude into the center of the cavity and are highly mobile. Older thrombi generally have smooth cavitary surfaces and they are less likely to change or embolize. In some cases vascularization and layer formation can be found.

Transthoracic echocardiography has a sensitivity of $90 \%$ - 95\% and a specificity of $85 \%$ - $95 \%$ for detection of LV Thrombus (LVT) in studies where the presence of thrombus was confirmed at surgery or autopsy [8] [9]. Echocardiographic criteria for LV Thrombus include:

- A distinct echogenic mass within the LV cavity (may be sessile/layered or protruding/mobile) that is contiguous with, but acoustically distinct from the underlying endocardial surface. It is seen throughout the cardiac cycle and visualized on at least two orthogonal views.

- An associated underlying region of severe wall motion abnormality, usually severe hypokinesis, akinesis, dyskinesis or aneurysmal dilatation.

Echocardiographically, left ventricular thrombus was defined as a discrete mass of echoes in the left ventricle that was distinct from the endocardium and seen in both systole and diastole, located in an area of asynergy, and identified in at least two views. Given the propensity for thrombi to form at the apex of the left ventricle, the best imaging planes to visualize LV thrombus are apical views, where the transducer is closest to the region of interest. The characteristics of the thrombus, including intracavitary motion, shape (protruding vs flat), echodensity, heterogeneity and central lucency as defined by Haugland, et al. [10] have been described. The shape of thrombus may be protruding or flat. Thrombi that projected into the ventricular cavity were classified as protruding and thrombi that did not were classified as flat. A thrombus has usually an echodensity similar to the myocardium while pannus appears more hyperechoic [11]. Figure 1 illustrates a large, organized, immobile, fibrosed and calcified thrombus which is flat along the endocardium of apical LV cavity and visible as horseshoeshaped in apical four chamber view of Transthoracic 2D echocardiographic imaging. An estimate of thrombus size, a one-dimensional measurement of maximal thrombus thickness was made perpendicular to the myocardium from the epicardial-pericardial interface to the innermost border of the thrombus-blood interface [12]. 
Figure 2 illustrates the maximal thrombus thickness of $18 \mathrm{~mm}$ in Tilted two chamber view. Certain normal anatomic structures (papillary muscle, false tendon, trabeculations) and technical artifacts (reverberation, near-field artifacts) will result in false positive diagnosis of LVT [13]. Figure 3 illustrates the tip of thrombus embedded along the papillary muscle in parasternal long axis view and a LV cavity dilation of $68 \mathrm{~mm}$ suggesting the dilated cardiomyopathy as a high risk factor for its development. High risk echocardiographic features for the development of LVT are shown in Table 1 given below.

In this patient severe global LV systolic dysfunction with a measured ejection fraction of $23 \%$ and LV dilatation of $68 \mathrm{~mm}$ were the echocardiographic risk factors for the development of LV Thrombus.

LV Thrombus may masquerade the conditions such as EMF (Endomyocardial fibrosis) and other cardiomyopathy syndromes with similar pathologies. Endomyocardial fibrosis is a "vanishing mystery" in the coastal districts of South India and the Kerala state was once "the hot spot” for this enigmatic disease [14], sub-endocardial fibrosis affecting the apices and the inflow tract of the right or left ventricle or both define the disease [15]. It is characterized by apical obliteration, valvular regurgitation especially the posterior leaflets of mitral or tricuspid valves, atrial dilation with features of restrictive physiology and the disease came to be known as "Davie's disease” [16].

A pedunculated thrombus in the left ventricular apex was visualized by the apical four chamber view of Transthoracic 2D echocardiography in dilated cardiomyopathy [17]. In the literature, there are two reports concerning the echocardiographic evidence of an intraventricular thrombus. Levisman et al. described the echocardiographic appearance of a pedunculated tumor in the left ventricle, which proved to be an organized thrombus [18]. De Joseph, et al. reported the echocardiographic findings in a patient with a large left ventricular thrombus [19]. In this patient, a large, flat, fibrosed, organized and calcified thrombus was visualized as horseshoe-shaped in apical four chamber, tilted two chamber and parasternal long axis views of 2D echocardiography in a 38 years old middle aged male with dilated cardiomyopathy.

\subsection{Embolic Risk}

Several studies have suggested that LV Thrombus that protrude into the ventricular cavity or that exhibit independent mobility are associated with a high risk of embolization. In dilated cardiomyopathy, the mechanism of embolization of LVT is less clear and several studies failing to demonstrate an increased risk of future embolization [20].

\subsection{Anticoagulation Therapy}

In patients with cardiomyopathy, the incidence of LV thrombus has been reported in the literature as $11 \%$ - $44 \%$ [21]. The definite treatment of LV thrombus is controversial. The main treatment options include thrombectomy, anticoagulation, and thrombolysis [22] [23].

Chronic oral anticoagulation has not been shown to decrease the risk of ischemic stroke or mortality in patients with LV dysfunction [24]. Anticoagulation should be individualized and considered in patients with a history of thromboembolic events, atrial fibrillation or evidence of an LV Thrombus. The level of anticoagulation

\begin{tabular}{cr} 
Table 1. High risk echocardiographic features for LVT. \\
\hline S. No & High risk echocardiographic features for the development of LVT \\
\hline 1 & Large infarct size and extent \\
2 & Anterior myocardial infarction $>>$ inferior infarction \\
3 & Severe global and regional LV systolic dysfunction, presence of CHF \\
4 & Elevated LV end-systolic volume, LV dilatation \\
5 & Spontaneous echo contrast \\
6 & Abnormal flow pattern within the LV \\
7 & Apical rotating flow \\
8 & Vortex ring formation \\
\hline
\end{tabular}


recommended varies but is generally targeted to an International Normalized Ratio (INR) of 2.0 to 3.0.

Similar to the post-myocardial infarction patient, a documented LVT in the setting of congestive heart failure and dilated cardiomyopathy likely warrants anticoagulation, especially if embolic potential is high and bleeding risk is low. Currently, no evidence exists for anticoagulation of chronic, layered, organized, mural thrombi. Due to the current lack of definitive studies, the primary prevention of cardio-embolic stroke through therapeutic anticoagulation remains controversial in patients with dilated cardiomyopathy and congestive heart failure. Despite the lack of supportive data, given that the greatest benefit would be expected for those with severe LV dysfunction (LVEF $<20 \%$ ) or a previous history of an embolic event, the use of anticoagulation in this select patient population may be reasonable [25].

In this patient, LV dilation of $68 \mathrm{~mm}$ with a LVEF of $23 \%$ and a documented LV Thrombus may suggest the use of anticoagulation is reasonable. Since the LV Thrombus in this case is a chronic, fibrosed, organized with calcification and densely adherent to the endocardium of LV apical region as a protective layer to limits its expansion and rupture, the benefit of anticoagulation is questionable. The patient may not experienced any embolic events previously and so the chronic anticoagulation therapy is not preferred in this case further after a trial of warfarin therapy for one month period.

\section{Conclusion}

Left ventricular thrombi in chronic dilated cardiomyopathy, visible as horseshoe-shaped, was detected by transthoracic 2D echocardiographic imaging. It is an interesting feature masquerading as endomyocardial fibrosis in a 38 years old middle aged man in coastal districts of South India at Thoothukudi region in Tamil Nadu state.

\section{References}

[1] Asinger, R.W., Mikell, F.L., Elsperger, J. and Hodges, M. (1981) Incidence of Left-Ventricular Thrombosis after Acute Transmural Myocardial Infarction. Serial Evaluation by Two-Dimensional Echocardiography. New England Journal of Medicine, 305, 297-302. http://dx.doi.org/10.1056/NEJM198108063050601

[2] Kalra, A. and Jang, I.K. (2000) Prevalence of Early Left Ventricular Thrombus after Primary Coronary Intervention for Acute Myocardial Infarction. Journal of Thrombosis and Thrombolysis, 10, 133-136. http://dx.doi.org/10.1023/A:1018710425817

[3] Greaves, S.C., Zhi, G., Lee, R.T., et al. (1997) Incidence and Natural History of Left Ventricular Thrombus Following Anterior Wall Acute Myocardial Infarction. American Journal of Cardiology, 80, 442-448. http://dx.doi.org/10.1016/S0002-9149(97)00392-5

[4] Nayak, D., Aronow, W.S., Sukhija, R., McClung, J.A., Monsen, C.E. and Belkin, R.N. (2004) Comparison of Frequency of Left Ventricular Thromb I in Patients with Anterior Wall versus Non-Anterior Wall Acute Myocardial Infarction Treated with Antithrombotic and Antiplatelet Therapy with or without Coronary Revascularization. American Journal of Cardiology, 93, 1529-1530. http://dx.doi.org/10.1016/j.amjcard.2004.02.066

[5] Ciaccheri, M., Castelli, G., Cecchi, F., et al. (1989) Lack of Correlation between Intracavitary Thrombosis Detected by Cross Sectional Echocardiography and Systemic Emboli in Patients with Dilated Cardiomyopathy. British Heart Journal, 62, 26-29. http://dx.doi.org/10.1136/hrt.62.1.26

[6] Gottdiener, J.S., Gay, J.A., VanVoorhees, L., DiBianco, R and Fletcher, R.D. (1983) Frequency and Embolic Potential of Left Ventricular Thrombus in Dilated Cardiomyopathy; Assessment by 2-Dimensional Echocardiography. American Journal of Cardiology, 52, 1281-1285. http://dx.doi.org/10.1016/0002-9149(83)90588-X

[7] Yamamoto, K., Ikeda, U., Furuhashi, K., et al. (1995) The Coagulation System Is Activated in Idiopathic Cardiomyopathy. Journal of American College of Cardiology, 25, 1634-1640. http://dx.doi.org/10.1016/0735-1097(95)00049-A

[8] Cacciapuoti, F., Varrichio, M., D’Avino, M., Gentile, S., Lama, D. and Cotrufo, M. (1986) Post Necrotic Endoventricular Thrombosis, Comparative Evaluation of the Diagnostic Reliability of 2-Dimensional Echocardiography and Cineventriculography. Gionale Italiano di Cardiologia, 16, 344-349.

[9] Srichai, M.B., Junor, C., Rodriguez, L.L., et al. (2006) Clinical, Imaging, and Pathological Characteristics of Left Ventricular Thrombus; a Comparison of Contrast-Enhanced Magnetic Resonance Imaging, Transthoracic Echocardiography and Transesophagial Echocardiography with Surgical or Pathological Validation. American Heart Journal, 152, 75-84. http://dx.doi.org/10.1016/j.ahj.2005.08.021

[10] Haugland, J.M., Asinger, R.W., Mikell, F.L., Elsperger, J. and Hodges, M. (1984) Embolic Potential of Left Ventricular Thrombi Detected by Two-Dimensional Echocardiography. Circulation, 70, 588-598.

http://dx.doi.org/10.1161/01.CIR.70.4.588 
[11] Mehra, S., Movahed, A., Espinoza, C. and Marcu, C.B. (2015) Horseshoe Thrombus in a Patient with Mechanical Prosthetic Mitral Valve. A Case Report and Review of Literature. World Journal of Clinical Cases, 3, 838-842. http://dx.doi.org/10.12998/wjcc.v3.i9.838

[12] Stratton, J.R. and Ritchie, J.L. (1984) The Effects of Antithrombotic Drugs in Patients with Left Ventricular Thrombi Assessment with Indium-111 Platelet Imaging and Two-Dimensional Echocardiography. Circulation, 69, 561. http://dx.doi.org/10.1161/01.CIR.69.3.561

[13] Asinger, R.W., Mikell, F.L., Sharma, B. and Hodges, M. (1981) Observations on Detecting Left Ventricular Thrombus with Two-Dimensional Echocardiography, Emphasis on Avoidance of False Positive Diagnoses. American Journal of Cardiology, 47, 145-156. http://dx.doi.org/10.1016/0002-9149(81)90303-9

[14] Sivasankaran, S. (2009) Restrictive Cardiomyopathy in India, the Story of a Vanishing Mystery. Heart, 95, 9-14. http://dx.doi.org/10.1136/hrt.2008.148437

[15] Williams, A.W., Ball, J.D. and Davies, J.N. (1954) Endomyocardial Fibrosis in Africa, Its Diagnosis, Distribution and Nature. Transactions of The Royal Society of Tropical Medicine and Hygiene, 48, 290-305. http://dx.doi.org/10.1016/0035-9203(54)90100-5

[16] Connor, D.H., Somers, K., Hutt, M.S., Manion, W.C. and D’Arbela, P.G. (1967) Endomyocardial Fibrosis in Uganda (Davies’ Disease). An Epidemiological, Clinical and Pathologic Study. American Heart Journal, Part-I, 74, 687-709. http://dx.doi.org/10.1016/0002-8703(67)90509-1

[17] Feigenbaum, H., Armstrong, W.F. and Ryan, T. (2005) Cardiomyopathies, Feigenbaum’s Echocardiography. 6th Edition, Chapter 17, 533.

[18] Levisman, J.A., MacAlpin, R.N., Abbasi, A.S., et al. (1975) Echocardiographic Diagnosis of a Mobile, Pedunculated Tumor in the Left Ventricular Cavity. American Journal of Cardiology, 36, 957-959. http://dx.doi.org/10.1016/0002-9149(75)90088-0

[19] De Joseph, R.L., Shiroff, R.A., Levenson, L.W., et al. (1977) Echocardiographic Diagnosis of Intraventricular Clot. Chest, 71, 417-419. http://dx.doi.org/10.1378/chest.71.3.417

[20] Katz, S.D. (1995) Left Ventricular Thrombus and the Incidence of Thromboembolism in Patients with Congestive Heart Failure, Can Clinical Factors Identify Patients at Increased Risk? Journal of Cardiovascular Risk, 2, 97-102. http://dx.doi.org/10.1097/00043798-199504000-00003

[21] Sharma, N.D., McCullough, P.A., Philbin, E.F. and Weaver, W.D. (2000) Left Ventricular Thrombus and Subsequent Thromboembolism in Patients with Severe Systolic Dysfunction. Chest, 117, 314-320. http://dx.doi.org/10.1378/chest.117.2.314

[22] Jeon, G.J., Song, B.G., Kim, T.H., Yang, H.J., Ma, B.O., Park, Y.H., et al. (2011) Catastrophic Massive Intracardiac Thrombus in a 40-Year-Old Patient with Dilated Cardiomyopathy. Nepal Medical College Journal, 13, 226-228.

[23] Nixon, J.V. (1983) Left Ventricular Mural Thrombus. Achieves Internal Medicine, 143, 1567-1571. http://dx.doi.org/10.1001/archinte.1983.00350080085018

[24] Homma, S. (2012) Warfarin and Aspirin in Patients with Heart Failure and Sinus Rhythm. New England Journal of Medicine, 366, 1859. http://dx.doi.org/10.1056/NEJMoa1202299

[25] Pulerwitz, T., Rabbani, I.F. and Pinney, S.P. (2004) A Rationale for the Use of Anticoagulation in Heart Failure Management. Journal of Thrombosis and Thrombolysis, 17, 87-93. http://dx.doi.org/10.1023/B:THRO.0000037663.81512.71 